\title{
Sugar Alcohols, Salt Stress, and Fungal Resistance: Polyols-Multifunctional Plant Protection?
}

\author{
John D. Williamson, ${ }^{1}$ Dianne B. Jennings, ${ }^{2}$ Wei-Wen Guo, ${ }^{3}$ and D. Mason Pharr ${ }^{4}$ \\ Department of Horticultural Science, North Carolina State University, Raleigh, NC 27695-7609 \\ Marilyn Ehrenshaft ${ }^{5}$ \\ Department of Botany, North Carolina State University, Raleigh, NC 27695-7612
}

\begin{abstract}
AdDitional INDEX WORDs. carbohydrates, oxidative stress, plant-pathogen interaction, polyols, transgenic plants
Abstract. The traditional use of polyols as osmotica in plant culture media is based on the assumption that polyols are not taken up or metabolized by cells. In reality, polyols are significant photosynthetic products and efficiently utilized metabolites in a large number of plants. In addition to these metabolic roles, initial interest in polyols focused primarily on their function as osmoprotectants. This was hypothesized to be due to their ability to act as compatible solutes. More recent research, however, indicates much broader roles for polyols in stress responses based on their significant antioxidant capacity. These include protection against salt and photooxidative stress as well as a potential role in plant pathogen interactions.
\end{abstract}

Many organisms including vascular plants synthesize and accumulate low molecular weight, soluble compounds in response to salt and drought stress (Loescher and Everard, 2000; Nuccio et al., 1999; Sakamoto and Murata, 2002; Stoopetal., 1996a). These compounds are often referred to as compatible solutes, because they can accumulate to high concentrations, but do not interfere (i.e., are compatible) with cell metabolism. One such class of compatible solutes is the sugar alcohols, or polyols, which are the chemically reduced forms of aldose or ketose sugars (Fig. 1). Although sucrose is probably the most widely studied plant carbohydrate, it has been estimated that up to $30 \%$ of all photosynthetically fixed carbon in plants is transported as polyols (Bieleski, 1982). Although polyols are sometimes the predominant form of translocated carbohydrate, they are always translocated in association with a sugar such as sucrose or raffinose. The most commonly reported polyols are the six-carbon sugar alcohols sorbitol, mannitol and galactitol (Fig. 1). Less commonly reported are seven-carbon polyols such as persitol (reduced mannoheptulose) which is found in avacado (Persea americana) (Liu et al., 1999), and volemitol (reduced sedoheptulose) which is found in Primula species such as cowslip (Primula veris) (Häflinger et al., 1999). Usually a single type of polyol is present in a plant species, and specific polyols are often characteristic of particular families or genera. For instance sorbitol (the reduced form of noncyclic glucose) commonly is found in the Rosaceae, where it constitutes an important translocated carbohydrate in apple (Malus domesticus) (Loescher et al., 1982), in Prunus species such as peach (Prunus persica), cherry and plum, and in its namesake genus Sorbus (mountain ash).

It has been suggested that polyols function as osmoprotectants, because the water-like hydroxyl groups in polyols allow them to form an artificial sphere of hydration around macromolecules, thus preventing metabolic inactivation under conditions of low osmotic

Received for publication 18 Dec. 2001. Accepted for publication 16 Apr. 2002. Sponsored by UDSA grants 9702011 and 9504552 to D.M.P. and J.D.W. and by the North Carolina Agricultural Research Service (NCARS), Raleigh. Use of trade names in this publication does not imply endorsement by the NCARS of products named, or criticism of similar ones not mentioned.

${ }^{1}$ Research associate professor; corresponding author; email john_williamson@ncsu.edu.

${ }^{2}$ Graduate student.

${ }^{3}$ Research analyst.

${ }^{4}$ Professor.

${ }^{5}$ Researcher. potential (Galinski and Trüper, 1994). Recently, however, there have been several reports that suggest polyols may play an even broader role in plant protection.

\section{Mannitol metabolism in plants}

One of the better-characterized sugar alcohols is mannitol, the chemically reduced form of mannose. Of the $>13$ different polyols isolated from higher plants (Bieleski, 1982; Lewis, 1984), mannitol may be the most widespread, being found in $>100$ species of plants in 70 diverse families as well as in bacteria and fungi (Jennings, 1984; Lewis and Smith, 1967; Stoop et al., 1996a). The potential roles of mannitol in celery as both an alternate carbon and energy source as well as an osmoprotectant have been reviewed in detail elsewhere (Loescher et al., 1995; Loescher and Everard, 2000; Nuccio et al., 1999; Pharr et al., 1995, 1999, Stoop et al., 1996a). Briefly, mannitol is synthesized in vascular plants from mannose-6-P through the action of a NADPH-mannose-6-P reductase (M6PR) (Fig. 2.). This enzyme catalyzes the conversion of mannose-6-P to mannitol 1-P, which is then dephosphorylated by a phosphatase to form mannitol (Loescher et al., 1992; Rumpho et al., 1983). Mannitol synthesis in celery occurs mainly in mature, source leaves where M6PR is localized in the cytosol of green palisade and spongy parenchyma tissues and bundle-sheath cells (Everard et al., 1993; Loescher et al., 1995). M6PR activity, and thus mannitol synthesis, is developmentally as well as light regulated, with high activities observed in mature leaves, but no detectable activities in sink tissues (such as roots and unstressed, immature leaves) (Everard et al., 1993, 1997 and Stoop and Pharr 1994). In extracts from mature leaves of celery plants irrigated with increasing concentrations of $\mathrm{NaCl}$ (up to $0.3 \mathrm{~m}$ ) there is little or no change in the amount of M6PR protein (Everard et al., 1994). Pulse-chase experiments using ${ }^{14} \mathrm{CO}_{2}$ in salt stressed celery, showed that these plants also retained essentially full mannitol biosynthetic capability, while water content and total photosynthetic capacity of the plant decreased substantially. The cDNA for M6PR has been cloned from celery leaf (Everard et al., 1997), and sequence analyses showed high similarity to a key enzyme in sorbitol biosynthesis in apple, aldose-6-P reductase (Kanayama et al., 1992) (see below). A final critical piece of the mannitol biosynthetic scheme was recently characterized by Gao and Loescher (2000), with the purification, characterization and cloning of the cDNA encoding 


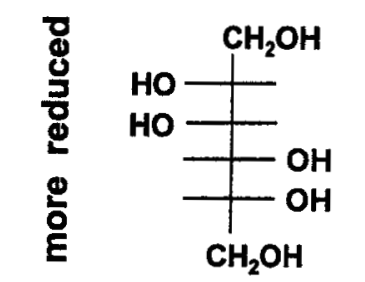

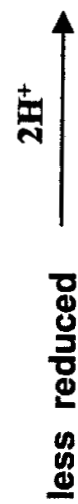

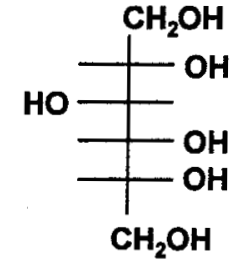

glucitol (sorbitol) (acyclic polyol)

vs.<smiles>CC(O)(C=O)C(C)(O)C(C)(O)C(C)(O)CO</smiles>

Glucose (acyclic aldose)

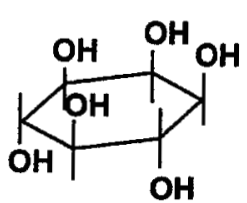

myo-inosotol (cyclic polyol)

vs.

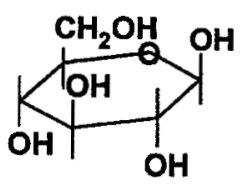

Glucopyranose (cyclic aldose)

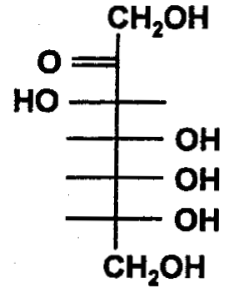

Sedoheptulose

vs.

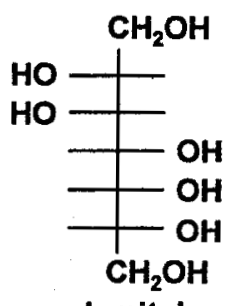

volemitol

Fig. 1. Chemical structures of some sugar alcohols and their corresponding sugars. Chemically reduced compounds (e.g., NADH) contain more energy than the analogous oxidized forms (e.g., $\mathrm{NAD}^{+}$). Hence, the sugar alcohols, being chemically more reduced, are higher energy compounds than their analogous sugars.

the celery cytosolic nonreversible glyceraldehyde-3-P dehydrogenase (nr-G3PDH). This enzyme provides the energy, in the form of NADPH, necessary for mannitol biosynthesis in celery leaves.

Mannitol use or catabolism is spatially separated from its synthesis (Fig. 2.). Mannitol synthesized in the leaf is translocated via phloem to sink tissues. To begin this journey, mannitol is apparently loaded into phloem by a proton symport mechanism mediated by a mannitol-specific transporter, mannitol transporter 1 (MAT-1) (Noiraud et al, 2001). Interestingly, the unusual mobility of the relatively immobile micronutrient B in polyol-containing plants has been linked to the formation of phloem translocated polyol-B

Fig. 2. Mannitol metabolism in celery. (Source) Biosynthesis via mannose-6-P reductase (M6PR). (Sink) Catabolic pathway via mannitol dehydrogenase (MTD). Other enzymes are phosphomannose isomerase (PMI), nonspecific sugar phosphatase (NSP) and hexokinase (HK). The phloem-localized, mannitol transporter (MAT1) is also indicated, and enzymes unique to mannitol metabolism are underlined. (Adapted from

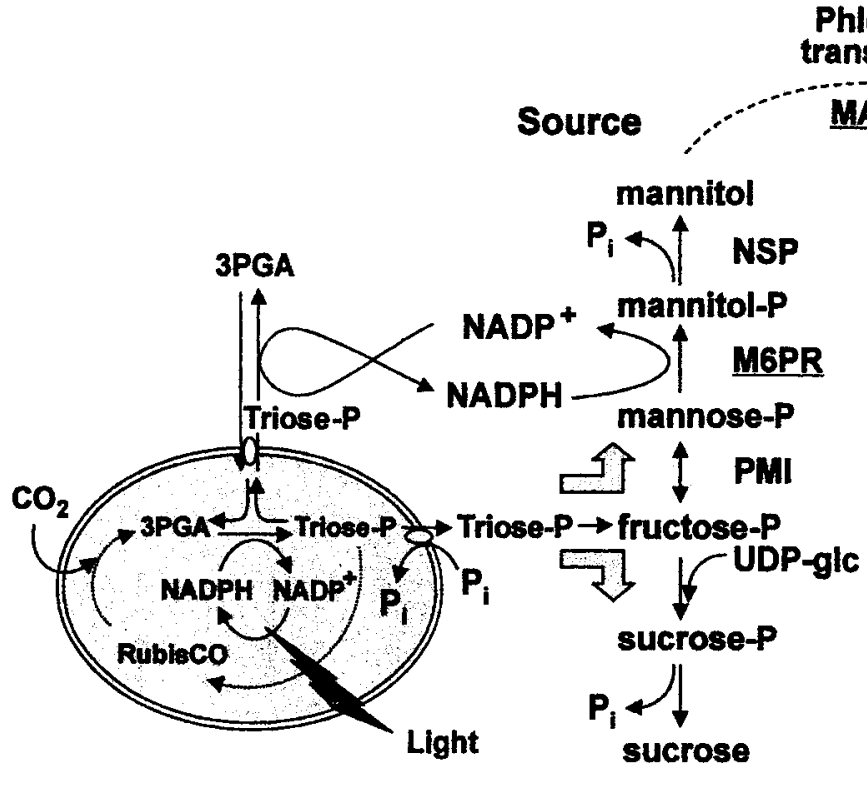

complexes (Hu et al., 1997). In celery, for instance, boron is translocated as a [mannitol:B:mannitol] complex.

Upon arrival in sink tissues, mannitol is catabolized through the action of the enzyme mannitol dehydrogenase (MTD). MTD provides the initial step by which translocated mannitol is committed to entry into the TCA cycle for use as a carbon and energy source (Pharr et al., 1995). Expression of MTD is repressed both by hexose sugars (Prata et al., 1997) and salt (Stoop et al., 1996a). As a result, under normal growth conditions plants may preferentially use sugars to maintain central metabolism. Sugar repression of mannitol utilization would allow large quantities of mannitol to be stored and function as reserve carbohydrate. When plants experience salt stress, MTD activity decreases further due to combined sugar suppression and salinity effects. This dual repression results in a further increase in mannitol accumulation. This type of regulation allows stressed plants that translocate both sucrose and mannitol to accumulate mannitol as an osmoprotectant rather than using it as an assimilate for growth, while at the same time they still have sucrose available for assimilation and growth. However, when energy and carbon demand are extremely high (e.g., in root and shoot meristems), sugars can be depleted and become limiting. Under these conditions, the resulting derepression of MTD can make the mannitol pool readily available as a carbon and energy source for maintenance of central metabolism. In celery, this type of regulation is reflected in the dramatic, differential mobilization of carbohydrate pools observed during the rapid reproductive growth characteristic of this biennial. Obaton (1929) observed that mannitol pools in celery began to decrease only after stored sugars fell below $1 \%$ of dry weight during flowering.

The integrated nature of mannitol metabolism in celery is further reflected, not only in the tight metabolic control of nrG3PDH described by Gao and Loescher 
(2000), but also by recent reports from Lemoine's group on the regulation of key celery carbohydrate transporters (Noiraud et al., $2000,2001)$. In celery, long-distance transport of reduced carbon occurs both in the form of sucrose (Suc) and mannitol, with the increased accumulation of mannitol correlating with increased resistance to salt stress. Noiraud et al. (2001) recently cloned the cDNA encoding the transporter that is apparently involved in phloem loading of mannitol, [Apium graveolens Mannitol Transporter (AgMaT-1)] (Fig. 2). Although Apium AgMAT-1 is undoubtedly a key player in mannitol utilization in celery, the cloning and characterization of key celery sucrose uptake transporters by this same group (Noiraud et al., 2000) more directly highlights the integration of metabolic processes. In this work they show that the high affinity Suc transporter, [AgSUT-1 (Apium graveolens Suc Uptake Transporter 1)] is mainly expressed in mature leaves and phloem of celery petioles, but also in sink organs such as roots. Most significantly, when celery plants were subjected to salt stress, AgSUT 1 expression decreased in all organs, but most markedly in roots, thus favoring accumulation of mannitol over sucrose.

\section{The cyclic polyol D-ononitol mediates osmotolerance}

The cyclic polyol D-(+) ononitol and its epimer D-pinitol are found in a several systematically unrelated plants ranging from ice plant (Mesembryanthemum crystallinum) to sugar pine (Pinus lambertiana) (Vernon and Bohnert, 1992 and references therein). Inositol (myo-inositol) is synthesized from glucose-6-P in two steps. First, the enzyme inositol phosphate synthase (INPS) catalyzes the conversion of glucose-6-P to inositol-1-P, which is then dephosphorylated by inositol monophosphatase (IMP). In M. crystallinum the stress-induced enzyme D-myo-inositol methyltransferase (IMT) then catalyses the methylation of myo-inositol to form D-ononitol (methyl-myo-inosotol) (Vernon and Bohnert, 1992). Although myoinositol

INPS
Glucose-6-P $\rightarrow$ inositol-1-P $\rightarrow$ myo-inositol $\rightarrow$ D-ononitol

and its derivatives are more commonly known for their involvement in cell signaling and membrane biogenesis, they also participate in osmotic stress responses. In ice plant, osmotic stress not only turns on expression of IMT, but also causes increased production of its substrate, myo-inositol. The resulting accumulation of D-ononitol has been proposed to not only facilitate sodium sequestration, but to also protect the photosynthetic apparatus from stress-induced increases in reactive oxygen species (ROS) (Nelson et al., 1998, 1999).

Although elegant proof of concept experiments by Tarczynski et al. $(1992,1993)$ demonstrated that transforming tobacco for cytosolic mannitol production conferred increased salt tolerance, levels of mannitol were low (1 to $2 \mu \mathrm{mol} \cdot \mathrm{g}^{-1}$ fresh weight). This is possibly due, among other possibilities, to the presence of an endogenous mannitol-catabolizing enzyme in tobacco (Jennings and Williamson, 1997, Jennings et al., 1998; Sheveleva et al., 1997a). In an attempt to circumvent this problem and produce plants with higher, more protective levels of polyols, Sheveleva et al. (1997b) transformed tobacco with an IMT coding sequence fused to the constitutive $35 \mathrm{~S}$ promoter. Although tobacco makes myo-inositol, it does not have a gene encoding IMT, and so does not make ononitol. An initial concern was that engineered production of ononitol might deplete endogenous myo-inositol pools. Rather unexpectedly, constitutive expression of IMT in tobacco led instead to the stress-induced accumulation of large amounts of ononitol $\left(36 \mu \mathrm{mol} \cdot \mathrm{g}^{-1}\right.$ fresh weight or $\approx 10 \mathrm{~mm}$ ). Analyses confirmed that there was no change in expression of the IMT transgene under stress conditions. Instead, osmotic stress appeared to induce the expression of an endogenous inositol-P-synthase (INPS), with a subsequent increase in synthesis of myo-inositol, the substrate for IMT-mediated production of ononitol.

In addition to its accepted role as an osmolyte, ononitol is an effective quencher of ROS (about as effective as mannitol, Orthen et al., 1994; Smirnoff, 1993; Smirnoff and Cumbes, 1989). Because D-ononitol was found in the chloroplast stroma as well as the cytosol of IMT transgenics, Sheveleva et al. (1997b) hypothesized it might protect the photosynthetic apparatus from increased photo-oxidative stress caused by low osmotic potential conditions. In fact, IMT transgenics suffered less inhibition of photosynthetic $\mathrm{CO}_{2}$ fixation during salt stress and drought, and recovered faster than wild type when returned to nonstressed conditions. Because myo-inositol, the substrate for IMT1, increases in tobacco under stress, preconditioning plants with permissive levels of $\mathrm{NaCl}$ further increased Dononitol production and resulted in increased protection when plants were subsequently transferred to high salt conditions. This work suggests that engineering stress-induced polyol accumulation might afford better protection than earlier constitutive osmolyte expression strategies.

\section{The polyol mannitol functions as an antioxidant in vivo}

The role of polyols as potent in vitro quenchers of (ROS) has been amply demonstrated (Orthenetal., 1994; Smirnoff and Cumbes, 1989; Smirnoff, 1993). As discussed above, it seems likely that polyols also function in this capacity in vivo. To test this hypothesis, Shen et al. (1997) targeted expression of a bacterial mannitol-1phosphate dehydrogenase gene to chloroplasts in transgenic tobacco by the addition of a plastid targeting sequence or transit peptide. The resulting transgenic tobacco accumulated much higher concentrations of mannitol than did tobacco expressing the same gene in the cytosol ( $7 \mathrm{vs} .1$ to $2 \mu \mathrm{mol} \cdot \mathrm{g}^{-1}$ fresh weight), while maintaining normal wild-type growth and photosynthetic performance under nonstress conditions. The accumulation of mannitol in chloroplasts $(\approx 100 \mathrm{mM})$ resulted in increased resistance to methyl viologen induced oxidative stress. In the presence of methyl viologen, isolated mesophyll cells of transgenic tobacco exhibited increased retention of chlorophyll as well as higher $\mathrm{CO}_{2}$ fixation rates than the wild type. When the hydroxyl radical probe dimethyl sulfoxide was introduced into cells, the initial formation rate of methane sulfinic acid was significantly lower in cells containing mannitol in the chloroplast than in wild-type cells, indicating an increased hydroxyl radical-scavenging capacity in transgenic tobacco. This suggests that the chloroplast-localized mannitol augments endogenous radical-scavenging mechanisms, thereby reducing oxidative cell damage. This is consistent with the report by Moore et al. (1997) that in the mannitol producers parsley and snapdragon, mannitol is almost equally distributed between the cytosol and chloroplasts.

\section{Sorbitol biosynthesis in transgenic tobacco: Too much of a good thing?}

In addition to the investigations of mannitol metabolism referenced above, there has been considerable research on the sugar alcohol sorbitol, the reduced form of glucose (Fig. 1). Sorbitol is a major photosynthetic product in several members of the family Rosaceae. As with mannitol, sorbitol accumulates in mature leaves, and is translocated to sink tissues (e.g., fruit and young leaves) (Bieleski, 1969). Sorbitol is formed from glucose-6-P via the action of NADP dependent sorbitol-6-P dehydrogenase (S6PDH) to form 

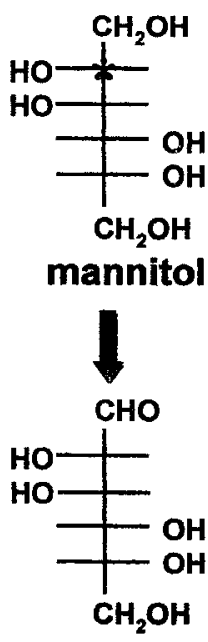

mannose

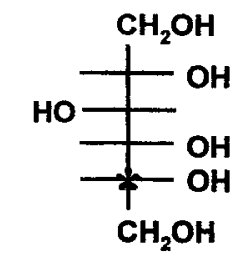

glucitol (sorbitol)

MTD

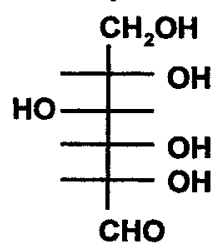

L-gulose

Fig 3. Stereospecificity of MTD oxidation of mannitol versus sorbitol. Stereospecific requirements for MTD substrates showed a marked preference for aldopentoses and aldo-hexoses with the same configuration as D-mannitol at carbon-2. (Stoop et al., 1996b). Chemically stated, the presence of a terminal diol with $2 \mathrm{R}$ chirality (*) appears to be the minimal requirement for alditols oxidized by MTD. Chirality at more distal carbons had little impact on acceptance of alditols as substrates. Thus D-glucitol (sorbitol) is oxidized, but to L-gulose. The oxidation of sorbitol to this nonmetabolizable aldose by MTD may be why they are rarely found together with mannitol in the same plant species.

sorbitol-6-P (S6P), which is then dephosphorylated by a phosphatase to yield sorbitol (Bieleski and Redgwell 1977; Hirai, 1981; Loescher et al., 1982; Yamaki, 1980). S6PDH has been purified from apple (Kanayama and Yamaki, 1993), the cDNA encoding the enzyme cloned (Kanayama et al., 1992), and its expression in transgenic tobacco shown to be sufficient for the synthesis of sorbitol (Tao et al., 1995). While these results implicate S6PDH as a key enzyme in the sorbitol biosynthetic pathway, levels of expression were extremely low.

If ectopic expression of polyol biosynthetic genes is universally efficacious, then one might expect a higher level of sorbitol expression in transgenic plants to be beneficial. A study by Sheveleva et al. (1998), however, demonstrates that this is not necessarily the case. In that study they examined 125 independent transgenic tobacco plants expressing Stpd1, the apple cDNA encoding S6PDH. Plants were identified that produced sorbitol in amounts ranging from 0.2 to $130 \mu \mathrm{mol} \cdot \mathrm{g}^{-1}$ fresh weight. Plants accumulating up to 2 to 3 $\mu \mathrm{mol} \cdot \mathrm{g}^{-1}$ fresh weight sorbitol appeared normal. However, plants accumulating higher levels of sorbitol showed progressively slower growth, became chlorotic and developed necrotic lesions. When sorbitol exceeded 15 to $20 \mu \mathrm{mol} \cdot \mathrm{g}^{-1}$ fresh weight, plants were infertile, and ultimately, at very high levels of sorbitol, were unable to form roots. Necrotic lesion formation correlated with high sorbitol, glucose, fructose, and starch, but low myo-inositol. Because lesion formation could be prevented by supplementing plant cultures with myo-inositol, Sheveleva et al. (1998) hypothesized that hyperaccumulation of sorbitol interfered with inositol biosynthesis, leading to osmotic imbalance and altered carbohydrate allocation and transport. Another possibility is suggested by the study of Stoop et al. (1996b), which showed that sorbitol (D-glucitol) can be oxidized by the mannitol catabolic enzyme MTD, but that the product is L-gulose rather than D-glucose (Fig. 3). With the exception of serving as an intermediate in vitamin C biosynthesis (Wheeler et al., 1998), L-gulose is not known to be metabolized by plants. It

seemed unlikely, therefore, that this reaction normally occurs in plants, and may be why sorbitol is not normally found together with mannitol in the same plant species. Jennings et al. (1998), however, demonstrated that, under certain conditions, even though tobacco does not have endogenous mannitol, significant levels of an endogenous MTD activity could be expressed in tobacco. If such plants had been engineered to produce sorbitol, the subsequent production of L-gulose might have unforeseen, deleterious effects.

\section{Mannitol, mannitol dehydrogenase, and plant-pathogen interaction}

Pathogen attack initiates a complex network of reactive oxygenmediated events in the plant (Alvarezet al., 1998). One of the earliest is the activation of an outer membrane localized enzyme, NADPH oxidase (Apostol et al., 1989; Keller et al., 1998), that catalyzes the massive production of ROS (e.g., superoxide and $\mathrm{H}_{2} \mathrm{O}_{2}$ ). ROS from this oxidative burst appear to serve several functions. First, the localized high concentration of ROS at the site of infection is directly antimicrobial. In addition, $\mathrm{H}_{2} \mathrm{O}_{2}$ is thought to signal a variety of complex cellular responses. At higher concentrations, $\mathrm{H}_{2} \mathrm{O}_{2}$ is hypothesized to trigger plant programmed cell death at the site of infection, thus limiting the spread of infection [hypersensitive response (HR)]. At lower concentrations, i.e., at a distance from the infection site, $\mathrm{H}_{2} \mathrm{O}_{2}$ is thought to be the signal that induces the production of the endogenous signal molecule salicylic acid (SA) (Sharma et al., 1996) that, in turn, induces a large group of defense genes collectively known as pathogenesis-related (PR) genes. This systemic induction of pathogen defense responses results in an acquired resistance to subsequent infection by the same or different pathogens [systemic acquired resistance (SAR)]. In addition to mediating induction of SAR responses, SA binds to and inactivates the antioxidant enzyme catalase (Chen et al., 1993), which converts $\mathrm{H}_{2} \mathrm{O}_{2}$ to $\mathrm{H}_{2} \mathrm{O}_{2} \mathrm{O}_{2}$. Inactivation of catalase presumably prevents $\mathrm{H}_{2} \mathrm{O}_{2}$ catabolism and thus maximizes $\mathrm{H}_{2} \mathrm{O}_{2}$-mediated defense responses.

Mannitol comprises up to $50 \%\left(\approx 60 \mu \mathrm{mol} \cdot \mathrm{g}^{-1}\right.$ fresh weight $)$ of the photosynthetically fixed, soluble carbohydrate in mature celery leaf (Loescher et al., 1992; Stoop and Pharr, 1994). As discussed above, mannitol is a potent antioxidant, and these large amounts of mannitol in celery might be expected to quench $\mathrm{H}_{2} \mathrm{O}_{2}$, and thus cripple $\mathrm{H}_{2} \mathrm{O}_{2}$-mediated defenses. However, Williamson et al. (1995, 1998) showed that not only is the celery MTD gene highly homologous to a pathogen-induced gene from parsley and Arabidopsis, but it is also induced by the PR-gene inducer, SA, even under normally repressive sugar levels. This suggested that, like the inactivation of catalase by SA, removal of mannitol via the induction of MTD could act to potentiate $\mathrm{H}_{2} \mathrm{O}_{2}$-mediated defenses in celery.

New research suggests that mannitol of microbial origin might also play a role in plant-pathogen interactions. In animals, defensive cells in the blood called killer leukocytes (also called neutrophylls or phagocytes), make ROS to destroy invading microbes (Rotrosen and Gallen, 1987). In turn, animal pathogens such as the fungus Cryptococus neoformans produce mannitol to quench this phagocytic active oxygen, and thus suppress this aspect of the organism's defenses (Chaturvedi et al., 1996). A similar suppression of active oxygen mediated plant defenses via the secretion of catalase by plant pathogenic bacteria has been hypothesized (Klotz and Hutcheson, 1992). It now appears that at least some fungal phytopathogens may use mannitol to suppress ROS-mediated plant defenses. Joosten et al. (1990) reported that pathogenic forms of the tomato pathogen Cladosporium fulvum produce mannitol, while mutants lacking the ability to produce mannitol were nonpathogenic. In addition, we 
have recently shown that mannitol production and secretion by a fungal pathogen of tobacco, Alternaria alternata, is massively induced by host plant extracts (Jennings et al., 1998). This same work, rather unexpectedly, revealed that although tobacco does not itself contain mannitol, it does have an endogenous, functional mannitol catabolic enzyme, MTD. Furthermore, MTD expression in tobacco appears to be a defense response, as MTD expression not only correlates with the presence of fungal infection, but is also induced by various chemical elicitors of PR-gene expression.

We hypothesized that this pathogen-induced expression of MTD provides a mechanism whereby the plant counters mannitol-mediated fungal suppression of PR responses by converting the fungal ROS quencher mannitol to mannose. To test this hypothesis we examined tobacco plants transformed to constitutively express a celery MTD, and found that constitutive MTD expression appeared to confer enhanced resistance to A. alternata, but not to the nonmannitol secreting fungal pathogen Cercospora nicotianae (Jennings, 2000). Together these findings not only raise interesting evolutionary questions, but also support the hypothesis that $M t d$, independent of its initially identified metabolic role in celery, represents a new class of pathogen resistance gene with potential utility for bioengineering fungal resistance in plants. Although we presently do not know how widespread this type of mannitol/MTD interaction might be, MTD homologs have been identified in at least two other plant genera. In addition to the pathogen-induced MTD homolog ELI-3 in Arabidopsis (Kiedrowski et al., 1992), MTD homologs have been identified in tomato (Lauter, 1996; van der Hoeven et al., 1999). Pathogen response of the tomato homolog was not examined, but, as tomato lacks mannitol, it seems possible that this putative MTD plays a role similar to that proposed for tobacco MTD.

\section{Polyols in parasitic plants}

Physiological and biochemical studies of parasitic plants have revealed some intriguing aspects of polyol metabolism that might be exploited in weed management. For example, it has been reported that several harmful parasitic-hemi-parasitic flowering plants, such as Orobanche sp. (Wegmann, 1986) Striga hermonthica (Stewart et al., 1984), and Thesium humile (Fer et al., 1993; Simier et al., 1998), that grow on a variety of warm climate crops contain the mannitol catabolic enzyme MTD, and use mannitol as a source of metabolic carbon. The proposed physiological roles of mannitol in these plants are similar to those proposed for celery, including storage of carbon and reducing power, osmoregulation and service as a compatible solute (Fer et al., 1993). However, two additional functions for mannitol in parasitic plants have been proposed (Smith et al., 1969). First, through osmotic adjustment, high levels of mannitol in the parasite might facilitate water movement from the host to the parasite. More significantly perhaps is the observation that, while these parasitic plants are able to convert host derived carbohydrates, such as sucrose, into mannitol, the host plants do not appear to take up or metabolize mannitol (Simier et al., 1998). Thus this could serve as a trapping mechanism for host photosynthate.

Given that the host plants do not appear to take up or metabolize mannitol, disruption of mannitol catabolism could be a promising target for selective chemical control of these weeds. In fact, stereochemical studies of substrate specificities showed that MTD has a distinct preference for aldopentoses and aldohexoses with the same configuration as D-mannitol at carbon-2 (Stoop et al., 1996b). As the rest of the molecule can be of variable structure, there is a potential to synthesize modified analogs of mannitol that inhibit MTD activity. These analogs could prove useful, not only in investigating the roles of mannitol catabolism in plants, but might also have promise as herbicides against mannitol-metabolizing plants such as the hemiparasitic weeds described above.

\section{Summary and Conclusions}

To date, most successful transgenic plant varieties have been engineered either by introduction of single gene traits (e.g., BT toxin) or through simple modifications in well-defined biochemical pathways (e.g., the FlavrSavr ${ }^{\mathrm{TM}}$ tomato). Many agronomic traits, however, are the result of complex genetic and physiological interactions, some of which are only beginning to be understood. For example, although production of polyols in tobacco provides increased osmoprotection, the ability to accumulate significant, protective levels of an introduced polyol can negatively impact growth, or worse. Thus, it may be desirable to devise strategies whereby polyols only accumulate under stress conditions. Although there may be several ways to achieve this (Nuccio et al., 1999), perhaps the most conceptually straightforward would be to use appropriately regulated plant promoters as well as the introduced ability to reutilize the carbon and energy sequestered in these compounds once stress is relieved. Suitable genes and promoters for this strategy are now becoming available, e.g., AgMtd, (Williamson et al., 1998) and Stpdl, (Bains et al., 1998).

\section{Literature Cited}

Alvarez, M.E., R.I. Pennell, P.J. Meijer, A. Ishikawa, R.A. Dixon, and C. Lamb. 1998. Reactive oxygen intermediates mediate a systemic signal network in the establishment of plant immunity. Cell 92:773-784.

Apostol, I., P.F. Heinstein, and P.S. Low. 1989. Rapid stimulation of an oxidative burst during elicitation of cultured plant cells. Role in defense and signal transduction. Plant Physiol. 90:109-116.

Bains, H.S., R. Tao, S.L. Uratsu, and A.M. Dandekar. 1998. Genomic nucleotide sequence of NADP sorbitol-6-phosphate dehydrogenase (NADPS6PDH) gene from apple (Accession No. AF057134). (PGR98-193) Plant Physiol. 118:1533.

Bieleski, R.L. 1969. Accumulation and translocation of sobitol in apple phloem. Austral. J. Biol. Sci. 22:611-620.

Bieleski, R.L. and R.J. Redgwell 1977. Synthesis of sorbitol in apricot leaves. Aust. J. Plant Physiol. 4:1-10.

Bieleski, R.L. 1982. Sugar alcohols, p. 158-192. In: F.A. Loewus and W. Tanner (eds.). Encyclopedia of plant physiology, new series. vol 13A. Springer-Verlag, New York.

Chaturvedi, V., B. Wong, and S.L. Newman. 1996. Oxidative killing of Cryptococcus neoformans by human neutrophils: Evidence that fungal mannitol protects by scavenging reactive oxygen intermediates. J. Immunol. 156:3836-3840.

Chen, Z., H. Silva, and D.F. Klessig. 1993. Active oxygen species in the induction of plant systemic acquired resistance by salicylic acid. Science 262:1883-1886.

Everard, J.D., V.R. Franceschi, and W.H. Loescher. 1993. Mannose-6phosphate reductase, a key enzyme in photoassimilate partitioning, is abundant and located in the cytosol of photosynthetically active cells of celery (Apium graveolens L.) source leaves. Plant Physiol. 102:345-356. Everard, J.D., R. Gucci, S.C. Kann, J.A. Flore, and W.H. Loescher. 1994. Gas exchange and carbon partitioning in the leaves of celery (Apium graveolens L.) at various levels of root zone salinity. Plant Physiol. 106:281-292.

Everard J.D., C. Cantini, R. Grumet, J. Plummer, and W.H. Loescher. 1997. Molecular cloning of mannose-6-phosphate reductase and its developmental expression in celery. Plant Physiol 113:1427-1435.

Fer, A., P. Simier, M.-C. Arnaud, L. Rey, and S. Renauidin. 1993. Carbon acquisition and metabolism in a root hemiparasitic angiosperm, Thesium humile (Santalaceae) growing on wheat (Triticum vulgare). Austral. J. Plant Physiol. 20:15-24. 
Galinski E.A. and H.G. Trüper. 1994. Microbial behavior in salt-stressed ecosystems. FEMS Microbiol. Rev. 15:95-108.

Gao, Z., and W.H. Loescher. 2000. NADPH supply and mannitol biosynthesis. Characterization, cloning, and regulation of the non-reversible glyceraldehyde-3-phosphate dehydrogenase in celery leaves. Plant Physiol. 124:321-330.

Häfliger, B., E.Kindhauser, and F. Keller. 1999. Metabolism of D-glycero-Dmanno-heptitol, volemitol, in Polyanthus. Discovery of a novel ketose reductase. Plant Physiol. 119:191-197.

Hirai, M. 1981. Purification and characteristics of sorbitol-6-phosphate dehydrogenase from loquat leaves. Plant Physiol. 67:221-224.

Hu, H., S.G. Penn, C.B. Lebrilla, and P.H. Brown. 1997. Isolation and characterization of soluble boron complexes in higher plants. The mechanism of phloem mobility of boron. Plant Physiol. 113:649-655.

Jennings, D.H. 1984. Polyol metabolism in fungi. Adv. Microb. Physiol. 25:149-193.

Jennings, D.B. 2000. The role of mannitol and mannitol dehydrogenase in plant-pathogen interactions. PhD diss. N.C. State Univ., Raleigh (online at http://www.lib.ncsu.edu/etd/).

Jennings, D.B. and J.D. Williamson. 1997. Induction of apparent mannitol dehydrogenase activity by INA in tobacco. Plant Physiol. 114:1150 (abstr.).

Jennings, D.B., M. Ehrenshaft, D.M. Pharr, and J.D. Williamson. 1998. Roles for mannitol and mannitol dehydrogenase in active oxygen mediated plant defense. Proc. Natl. Acad. Sci. USA 95:15129-15133.

Joosten, M.H.A.J., L.J.M. Hendrickx, and P.J.G.M. de Wit. 1990. Carbohydrate composition of apoplastic fluids isolated from tomato leaves inoculated with virulent or avirulent races of Cladosporium fulvum. Neth. J.Plant Pathol. 96:103-112.

Kanayama, Y., H. Mori, H. Imaseki, and S. Yamaki 1992. Nucleotide sequence of a cDNA encoding sorbitol-6-phosphate dehydrogenase from apple. Plant Physiol. 100:1607-1608.

Kanayama, Y. and S. Yamaki 1993. Purification and properties of NADPdepedent sorbitol-6-phosphate dehydrogenase from apple seedlings. Plant Cell Physiol. 34:819-823.

Keller, T., H.G. Damude, D. Werner, P. Doerner, R.A. Dixon, and C. Lamb. 1998. A plant homolog of the neutrophil NADPH oxidase gp9lphox subunit gene encodes a plasma membrane protein with $\mathrm{Ca}^{2+}$ binding motifs. Plant Cell 10:255-266.

Kiedrowski, S., P. Kawalleck, K. Hahlbrock, I.E. Somssich, and J.L. Dangl. 1992. Rapid activation of a novel plant defense gene is strictly dependent on the Arabidopsis RPM1 disease resistance locus. EMBO J. 11:46774684.

Klotz, M.G. and S.W. Hutcheson. 1992. Multiple periplasmic catalases in phytopathogenic strains of Pseudomonas syringae. Appl. Environ. Microbiol. 58:468-473.

Lauter, F.R. 1996. Root-specific expression of the LeRse-1 gene in tomato is induced by exposure of the shoot to light. Mol. Gen. Genet. 252:751-754.

Lewis, D.H. 1984. Physiology and metabolism of alditols, p. 157-179. In: D.H. Lewis (ed.). Storage carbohydrates in vascular plants . Cambridge Univ. Press, Cambridge, U.K.

Lewis, D.H. and D.C. Smith. 1967. Sugar alcohols (polyols) in fungi and green plants. I. Distribution, physiology and metabolism. New Phytol. 66:143-184.

Liu, X., P.W. Robinson, M.A. Madore, G.W.Witney, and M.L. Arpaia. 1999. 'Hass' avacado carbohydrate fluctuations. II. Fruit growth and ripening. J. Amer. Soc. Hort. Sci. 124:676-681.

Loescher, W.H., G.C. Marlow, and R.A. Kennedy. 1982. Sorbitol metabolism and sink-source interconversions in developing apple leaves. Plant Physiol. 70:335-339.

Loescher, W.H., R. H. Tyson, J.D. Everard, R.J. Bagwell, and R.L. Bieleski. 1992. Mannitol synthesis in higher plants. Plant Physiol. 98:1396-1420.

Loescher, W.H., J.D. Everard, C. Cantini, and R. Grumet. 1995. Sugar alcohol metabolism in source leaves in carbon partitioning and source-sink interactions in plants, p. 170-179. In: M.A. Madora and W.J. Lewis (eds.). Current topics in plant physiology. vol 13. Amer. Soc. Plant Physiolo., Wash., D.C.
Loescher, W.H. and J.D. Everard. 2000. Regulation of sugar alcohol biosynthesis, p. 275-299. In: R.C. Leegood, T.D. Sharkey, and S. von Caemmerer (eds.). Photosynthesis: Physiology and metabolism. Kluwer Academic Pubs., Dordrecht, The Netherlands.

Moore, B., D. Palmquist, and J.R. Seemann. 1997. Influence of plant growth at high $\mathrm{CO}_{2}$ concentrations on leaf content of ribulose-1,5-bisphosphate carboxylase/oxygenase and intracellular distribution of soluble carbohydrates in tobacco, snapdragon and parsley. Plant Physiol. 115:241-248.

Nelson, D.E., G. Rammesmayer, and H.J. Bohnert. 1998. Regulation of cellspecific inositol metabolism and transport in plant salinity tolerance. Plant Cell 10:753-764.

Nelson, D.E., M. Koukoumanos, and H.J. Bohnert 1999. Myo-inositoldependent sodium uptake in ice plant. Plant Physiol. 119:165-172.

Noiraud, N., S. Delrot, and R. Lemoine. 2000. The sucrose transporter of celery. Identification and expression during salt stress. Plant Physiol. 122:1447-1456.

Noiraud, N., L. Maurousset, and R. Lemoine 2001. Identification of a mannitol transporter, AgMaT1, in celery phloem. Plant Cell 13:695-705.

Nuccio, M.L., D. Rhodes, S.D. McNeil, and A.D. Hanson. 1999. Metabolic engineering of plants for osmotic stress resistance. Current Opinions Plant Biol. 2:128-134.

Obaton, M.F. (1929) Evolution del la mannite (mannitol) chez les vegetaux. Rev. Gen. Bot. (Revue de Botanique) 41:622-633.

Orthen, B., M. Popp, and N. Smirnoff. 1994. Hydroxyl radical scavenging properties of cyclitols. Proc. Royal Soc. Edinburgh. B. Biol. Sci. 102:269272.

Pharr, D.M., J.M.H. Stoop, J.D. Williamson, M.E. Studer Feusi, M.O. Massel, and M.A Conkling. 1995. The dual role of mannitol as osmoprotectant and photoassimilate in celery. HortScience 30:1182-1188.

Pharr, D.M., R.T.N. Prata, D.B. Jennings, J.D. Williamson, E. Zamski, Y.T. Yamamoto, and M.A. Conkling. 1999. Regulation of mannitol dehydrogenase: relationship to plant growth and stress tolerance. HortScience 34:10271032.

Prata, R.T.N., J.D. Williamson, M.A. Conkling, and D.M. Pharr. 1997. Sugar repression of mannitol dehydrogenase activity in celery cells. Plant Physiol. 114:307-314.

Rotrosen, D. and J.I. Gallen 1987. Disorders of phagocyte function. Annu. Rev. Immunol. 5:127-150.

Rumpho, M.E., G.E. Edwards, and W.H. Loescher. 1983. A pathway for photosynthetic carbon flow to mannitol in celery leaves. Activity and localization of key enzymes [Apium graveolens]. Plant Physiol. 73:869873.

Sakamoto, A. and N. Murata. 2002. The role of glycine betaine in the protection of plants from stress: Clues from transgenic plants. Plant Cell Environ. 25:163-171.

Sharma, Y.K., J. Leon, I. Raskin, and K.R. Davis. 1996. Ozone-induced responses in Arabidopsis thaliana: The role of salicylic acid in the accumulation of defense-related transcripts and induced resistance. Proc. Natl. Acad. Sci. USA 93:5099-5104.

Shen, B., R.G. Jensen, and H.J. Bohnert. 1997. Increased resistance to oxidative stress in transgenic plants by targeting mannitol biosynthesis to chloroplasts. Plant Physiol. 113:1177-1183.

Sheveleva, E.V., H.J. Bohnert, and R.G. Jensen. 1997a. Metabolic engineering of polyol production in Nicotiana tabacum. Plant Physiol. (Suppl.)114:298 (abstr. 1559).

Sheveleva, E.V., W. Chmara, H.J. Bohnert, and R.G. Jensen. 1997b. Increased salt and drought tolerance by D-ononitol production in transgenic Nicotiana tabacum. Plant Physiol. 115:1211-1219.

Sheveleva, E.V., S. Marquez, W. Chmara, A. Zegeer, R.G. Jensen, and H.J. Bohnert. 1998. Sorbitol-6-phosphate dehydrogenase expression in transgenic tobacco. High amounts of sorbitol lead to necrotic lesions. Plant Physiol. 117:831-839.

Simier, P., S. Robert, and A. Fer. 1998. Mannitol metabolism in darkness in the leaves of the hemiparasitic angiosperm, Thesium humile. Plant Physiol. Biochem. 36:237-245.

Smirnoff, N. and Q.J. Cumbes. 1989. Hydroxyl radical scavenging activity of compatible solutes. Phytochemistry 28:1057-1060. 
Smirnoff, N. 1993. Tansley review No. 52: The role of active oxygen in the response of plants to water stress and desiccation. New Phytol. 125:27-58.

Smith, D., L. Muscatine, and D. Lewis. 1969. Carbohydrate movement from autotroph to hetrotrophs in parasitic and mutualistic symbosis. Biol. Rev. 44:17-90.

Stewart, G.R., J. Nour, M. MacQueen, and N. Shah. 1984. Aspects of the biochemistry of Striga, p. 161-178. In: Ayensu, E.S., H. Dogett, R.D. Keynes, J.M. Laferve, L. Musselmann, C. Parker, and A. Pickering (eds.). Striga biology and control. ICSU Press, Paris.

Stoop, J.M.H. and D.M. Pharr. 1994. Mannitol metabolism in celery stressed by excess macronutrients. Plant Physiol. 106:503-511.

Stoop, J.M.H., J.D. Williamson, and D.M. Pharr. 1996a. Mannitol metabolism in plants: a method for coping with stress. Trends Plant Sci. 1:139144.

Stoop, J.M.H., W.S. Chilton, and D.M. Pharr. 1996b. Substrate stereospecificity of the NAD-dependent mannitol dehydrogenase from celery. Phytochemistry 43:1145-1150.

Tao, R., S.L. Uratsu, and A.M. Dandekar 1995. Sorbitol synthesis in transgenic tobacco with apple cDNA encoding NADP-dependent sorbitol6-phosphate dehydrogenase. Plant Cell Physiol. 36:525-532.

Tarczynski, M.C., R.G. Jensen, and H.J. Bohnert. 1992. Expression of a bacterial $m t l D$ gene in transgenic tobacco leads to production and accumulation of mannitol. Proc. Natl. Acad. Sci. USA 89:2600-2604.

Tarczynski, M.C., R.G. Jensen, and H.J. Bohnert. 1993. Stress protection of transgenic tobacco by production of the osmolyte mannitol. Science 259:508-510.

van der Hoeven, R.S., A.L. Matern, T. Vision, I.E. Holt, F. Liang, J. Upton,
C.M. Ronning, M.B. Craven, C.Y. Fujii, C.L. Bowman, W. Nierman, C.M. Fraser, J.C. Venter, G.B. Martin, J.J. Giovannoni, and S.D. Tanksley. 1999. Generation of ESTs from tomato shoot meristem. GeneBank Accession AI490847.

Vernon, D.M. and H.J. Bohnert. 1992. A novel methyl transferase induced by osmotic stress in the facultative halophyte Mesembryanthemum crystallinum. EMBO J. 11:2077-2085.

Wegmann, K. 1986. Biochemistry of osmoregulation and possible biochemical reasons of resistance against Orobanche, p. 107-113. In: S.J. ter Borg (ed.). Biology and control of Orobanche: Proceedings of a workshop in Wageningen, The Netherlands.

Wheeler, G.L., M.A. Jones, and N. Smirnoff. 1998. The biosynthetic pathway of vitamin $C$ in higher plants. Nature 393:365-369.

Williamson, J.D., J.M.H. Stoop, M.O. Massel, M.A. Conkling, and D.M. Pharr. 1995. Sequence analysis of a mannitol dehydrogenase cDNA from plants reveals a function for the PR protein ELI3. Proc. Natl. Acad. Sci. USA 92:7148-7152.

Williamson, J.D., W.-W. Guo, and D.M. Pharr. 1998. Cloning and characterization of a genomic clone (accession no. AF067082) encoding mannitol dehydrogenase, a salt, sugar and SA regulated gene from celery (Apium graveolens L.) (\#PGR98-137). Plant Physiol. 118:329.

Yamaki, S. 1980. Properties of sorbitol-6-phosphate dehydrogenase and its connection with sorbitol accumulation in apple. HortScience. 15:268-270.

Zamski, E., W.-W. Guo, Y.T. Yamamoto, D.M. Pharr, and J.D. Williamson. 2001. Analysis of celery (Apium graveolens) mannitol dehydrogenase $(M t d)$ promoter regulation in Arabidopsis suggests roles for MTD in key environmental and metabolic responses. Plant Mol. Biol. 47:621-631. 